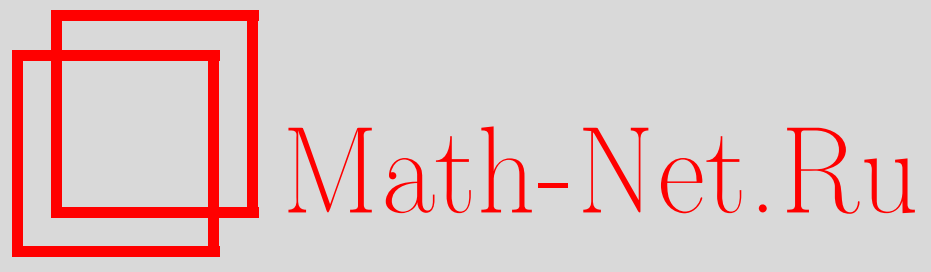

И. А. Пятышев, Операции над аппроксимативно компактными множествами, Матем. заметки, 2007, том 82, выпуск 5, 729-735

DOI: https://doi.org/10.4213/mzm4085

Использование Общероссийского математического портала Math-Net.Ru подразумевает, что вы прочитали и согласны с пользовательским соглашением http://www . mathnet.ru/rus/agreement

Параметры загрузки:

IP: 3.80 .181 .102

26 апреля 2023 г., 02:42:13

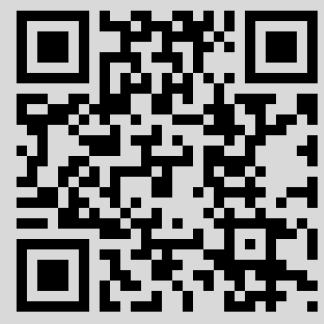


Том 82 выпуск 5 ноябрь 2007

УДК 517.982.256

\section{Операции над аппроксимативно компактными множествами}

\section{И. А. Пятышев}

В работе рассматривается вопрос сохранения свойства аппроксимативной компактности при различных операциях. В произвольном равномерно выпуклом сепарабельном пространстве построен пример двух аппроксимативно компактных множеств, пересечение которых не аппроксимативно компактно. Построен пример двух линейных аппроксимативно компактных множеств, замыкание алгебраической суммы которых не аппроксимативно компактно. В произвольном банаховом пространстве построены два нелинейных аппроксимативно компактных множества, алгебраическая сумма которых замкнута, но не аппроксимативно компактна. Доказано также, что в любом равномерно выпуклом банаховом пространстве существует аппроксимативно компактная каверна.

Библиография: 8 названий.

Введение. Пусть $M$ - некоторое подмножество банахова пространства $X$. Последовательность $\left\{y_{n}\right\}_{n=1}^{\infty} \subset M$ называется минимизирующей для элемента $x \in X$, если $\left\|y_{n}-x\right\| \rightarrow \rho(x, M):=\inf \{\|x-y\|: y \in M\}$ при $n \rightarrow \infty$.

ОПРЕДЕЛЕНИЕ 1. Множество $M$ аппроксимативно компактно, если для любого $x \in X$ всякая минимизирующая последовательность содержит подпоследовательность, сходящуюся к элементу из $M$.

Понятие аппроксимативно компактного множества было введено Ефимовым и Стечкиным [1] и играет важную роль в теории приближений в нормированных пространствах [2]. В то же время само свойство аппроксимативной компактности исследовано сравнительно мало.

Цель настоящей работы - выяснить, сохраняется ли свойство аппроксимативной компактности при различных операциях. По ходу дела строятся новые примеры аппроксимативно компактных множеств.

Приведем некоторые сведения об аппроксимативно компактных множествах, необходимые для дальнейшего.

Всякое аппроксимативно компактное множество $M$ замкнуто, а также является множеством существования, т.е. для любого $x \in X$ существует элемент наилучшего приближения $y \in M:\|x-y\|=\rho(x, M)$.

Очевидно, всякое ограниченно компактное множество (т.е. множество, пересечение которого с каждым замкнутым шаром компактно) является аппроксимативно

Работа выполнена при поддержке Российского фонда фундаментальных исследований (грант № 05-01-00962).

(C) И. А. Пятышев, 2007 
компактным. Неизвестно, в каждом ли банаховом пространстве существует аппроксимативно компактное, но не ограниченно компактное множество. В любом сепарабельном банаховом пространстве существует ограниченное аппроксимативно компактное, но не компактное множество [3] (соответствующий пример описан ниже в доказательстве теоремы 1).

В достаточно "хороших" пространствах $X$ аппроксимативно компактными являются все выпуклые замкнутые множества. Такие пространства называются пространствами Ефимова-Стечкина.

ОПРЕДЕЛЕНИЕ 2. Банахово пространство $X$ называется пространством Ефимова-Стечкина, если для любой последовательности $\left\{x_{n}\right\} \subset X,\left\|x_{n}\right\|=1$, из того, что $f \in X^{*},\|f\|=1, f\left(x_{n}\right) \rightarrow 1$, следует существование у $\left\{x_{n}\right\}$ сходящейся подпоследовательности.

В работе Зингера [4] доказана

Теорема А. Следующие условия эквивалентны:

1) $X$ - пространство Ефимова-Стечкина;

2) каждое выпуклое замкнутое множество в $X$ аппроксимативно компактно.

Другие характеристики пространств Ефимова-Стечкина см. в [5]. Примером пространства Ефимова-Стечкина служит любое равномерно выпуклое пространство (определение см. ниже), в частности, любое пространство $L_{p}, 1<p<\infty$.

Теоретико-множественные операции над аппроксимативно компактными множествами. Нетрудно заметить, что объединение $M$ любого конечного числа аппроксимативно компактных множеств $M_{1}, \ldots, M_{n}$ также аппроксимативно компактно. Счетное объединение аппроксимативно компактных множеств уже может не быть аппроксимативно компактным: например, одноточечные множества на единичной сфере, объединение которых не компактно.

Понятно, что разность двух аппроксимативно компактных множеств может не оказаться аппроксимативно компактной уже потому, что она, вообще говоря, не является замкнутым множеством. Но и замыкание этой разности может не быть аппроксимативно компактным, как показывает пример разности двух замкнутых концентрических шаров в произвольном пространстве Ефимова-Стечкина.

Что касается пересечения, то, например, пересечение двух выпуклых замкнутых (а значит, аппроксимативно компактных в силу теоремы А) множеств в пространстве Ефимова-Стечкина тоже выпукло и замкнуто, а следовательно, аппроксимативно компактно. В произвольном банаховом пространстве это неверно: можно построить пример двух аппроксимативно компактных гиперплоскостей в пространстве $l_{1}$, пересечение которых не аппроксимативно компактно.

Покажем, что аппроксимативная компактность не сохраняется при пересечении даже в самых "хороших" пространствах.

ОПРЕДЕЛЕНиЕ 3. Следуя [2], скажем, что банахово пространство $X \in(C L U R)$, если из $\|x\|=\left\|x_{n}\right\|=1,\left\|x+x_{n}\right\| \rightarrow 2, n \rightarrow \infty$, вытекает существование сходящейся подпоследовательности $x_{n_{k}}$.

В класс $(C L U R)$ входят, в частности, все равномерно выпуклые банаховы пространства, т.е. пространства со свойством: для любого $\varepsilon>0$ существует такое $\delta>0$, что из соотношений $\|x\|=\|y\|=1,\|x-y\|>\varepsilon$ следует неравенство $\|x+y\| / 2<1-\delta$. 
Теорема 1. В любом сепарабельном банаховом пространстве $X \in(C L U R)$ существуют два аппроксимативно компактных множества, пересечение которых не аппроксимативно компактно.

ДокАЗАТЕЛЬСтво. Построим в $X$ два аппроксимативно компактных множества $M_{1}$ и $M_{2}$, пересечение которых не аппроксимативно компактно.

Обозначим через $B$ открытый единичный шар в $X$. Положим $M_{1}=(X \backslash B) \cup\{0\}$. Приведем лемму 1.3 из [2].

Для $M=X \backslash B$ эквивалентны следующие условия:

а) $X \in(C L U R)$

б) для любого $x \in B \backslash\{0\}$ и для любой последовательности $y_{n} \in X \backslash B$ из $\left\|y_{n}-x\right\| \rightarrow \rho(x, X \backslash B), n \rightarrow \infty$, следует существование сходящейся подпоследовательности $y_{n_{k}}$.

Из этой леммы легко доказать, что $M_{1}$ аппроксимативно компактно: для любого элемента $x$ минимизирующая последовательность из $M_{1}$ либо постоянна и состоит из одного нулевого элемента, либо содержит минимизирующую для $x$ подпоследовательность из $X \backslash B$.

Теперь построим второе аппроксимативно компактное множество $M_{2}$. Рассмотрим две последовательности $\left\{x_{n}\right\}_{n=1}^{\infty} \subset X,\left\{y_{n}\right\}_{n=1}^{\infty} \subset X$ со следующими свойствами: $\left\|x_{n}\right\|=\left\|y_{n}\right\|=1$, последовательность $\left\{y_{n}\right\}$ всюду плотна на единичной сфере пространства $X, \lim \inf \left\|x-x_{n}\right\| \geqslant\|x\|$ для любого $x$. Положим

$$
M_{2}=\left(\{0\} \cup\left\{\frac{y_{n}}{10 n}\right\} \cup\left\{10 x_{n}\right\}\right)+v,
$$

где $v$ - некоторый элемент с $\|v\|=1 / 2$.

В работе [3] доказано существование последовательности $\left\{x_{n}\right\}$ в произвольном сепарабельном пространстве и аппроксимативная компактность множества $M_{2}$.

Из последовательности $\left\{x_{n}\right\}$ нельзя выбрать сходящуюся подпоследовательность. Действительно, если есть некоторая сходящаяся подпоследовательность $x_{n_{k}} \rightarrow x$, то

$$
\|x\| \leqslant \liminf \left\|x-x_{n}\right\| \leqslant \liminf \left\|x-x_{n_{k}}\right\|=0,
$$

откуда $\|x\|=0$, что противоречит равенству $\left\|x_{n_{k}}\right\|=1$.

Имеем

$$
\left(\left(\{0\} \cup\left\{\frac{y_{n}}{10 n}\right\}\right)+v\right) \cap M_{1}=\varnothing, \quad\left(\left\{10 x_{n}\right\}+v\right) \cap M_{1}=\left\{10 x_{n}\right\}+v,
$$

а следовательно, $M=M_{1} \cap M_{2}=\left\{10 x_{n}\right\}+v$. Для элемента $v$ последовательность $\left\{10 x_{n}+v\right\}$ является минимизирующей на $M$, но, как уже отмечалось, из этой последовательности нельзя выбрать сходящуюся подпоследовательность. Следовательно, $M$ не аппроксимативно компактно.

Теорема 1 доказана.

Было бы интересно проверить, справедливо ли утверждение теоремы 1 для пространств Ефимова-Стечкина. 
Аппроксимативно компактная каверна. Каверной назовем замыкание дополнения к выпуклому телу.

Замечание 1 . В любом банаховом пространстве $X \in(C L U R)$ существует аппроксимативно компактная каверна.

ДоказАтельство. Возьмем множество $M=X \backslash(B \cap(B+v))$, где $v$ - произвольный элемент с $\|v\|=1 / 2$. Очевидно, что $M$ является каверной. Его аппроксимативная компактность легко следует из леммы 1.3 работы [2] (см. ее формулировку в доказательстве теоремы 1). Возьмем произвольный элемент $x \notin M$. В случае $x \neq 0, x \neq v$ минимизирующая для $x$ из $M$ последовательность $\left\{w_{n}\right\}$ вкладывается в объединение $\left\{x_{n}\right\} \cup\left\{y_{n}\right\}$, где $\left\{x_{n}\right\}$ - некоторая минимизирующая для $x$ последовательность из $X \backslash B,\left\{y_{n}\right\}$ - некоторая минимизирующая для $x$ последовательность из $X \backslash(B+v)$. Поскольку $x$ не является центром для шаров $B, B+v$, по лемме 1.3 из $\left\{x_{n}\right\},\left\{y_{n}\right\}$ можно выбрать сходящиеся подпоследовательности, а следовательно, из $\left\{w_{n}\right\}$ можно выбрать сходящуюся подпоследовательность. В случае $x=0$ имеем $\left\{w_{n}\right\} \subset\left\{y_{n}\right\} ;$ в случае $x=v$ имеем $\left\{w_{n}\right\} \subset\left\{x_{n}\right\}$, начиная с некоторого номера. Поскольку $x=0$ не является центром шара $B+v$, а $x=v$ не является центром шара $B$, по лемме 1.3 из $\left\{x_{n}\right\},\left\{y_{n}\right\}$ можно выбрать сходящиеся подпоследовательности, а следовательно, из $\left\{w_{n}\right\}$ можно выбрать сходящуюся подпоследовательность.

Алгебраическая сумма аппроксимативно компактных множеств. Алгебраической суммой двух множеств $M_{1}, M_{2}$ называется множество

$$
M_{1}+M_{2}=\left\{x=y+z: y \in M_{1}, z \in M_{2}\right\} .
$$

Алгебраическая сумма двух аппроксимативно компактных множеств может быть не аппроксимативно компактной уже потому, что она, вообще говоря, не является замкнутым множеством: в [6; задача № 273, с. 225 , решение на с. 325$]$ приводится пример двух замкнутых подпространств в гильбертовом пространстве, алгебраическая сумма которых незамкнута. С другой стороны, замыкание суммы двух подпространств в гильбертовом пространстве (как и в любом пространстве Ефимова-Стечкина) аппроксимативно компактно.

Построим два аппроксимативно компактных подпространства в пространстве $L_{1}$, алгебраическая сумма которых замкнута, но не аппроксимативно компактна.

Пусть $L_{1}(K, \mu)$ - пространство действительнозначных функций, определенных на множестве $K$ произвольной природы и суммируемых на $K$ по мере $\mu$, определенной на некоторой $\sigma$-алгебре подмножеств этого множества. Пусть множество $K$ разбито на два непересекающихся измеримых множества $K_{1}$ и $K_{2}$, и имеется линейный оператор $A: L_{1}\left(K_{1}\right) \rightarrow L_{1}\left(K_{2}\right)$ с нормой $\|A\|<1$. Рассмотрим подпространство

$$
Y_{A}=\left\{y \in L_{1}(K):=\left.y\right|_{K_{2}}=A\left(\left.y\right|_{K_{1}}\right)\right\} .
$$

Лемма 1. Подпространство $Y_{A}$ аппроксимативно компактно в $L_{1}(K, \mu)$.

ДокАзАтЕЛЬСтво. Будем представлять каждую функцию $f$ из $L_{1}(K)$ как пару функций $(x, y)$, где $x$ - сужение $f$ на множество $K_{1}$, а $y$ - сужение $f$ на множество $K_{2}$. Функциям из подпространства $Y_{A}$ соответствуют пары $(x, A(x))$. Известно (см. замечание 2 к теореме 1 в [7]), что расстояние от пары $(x, y)$ до $Y_{A}$ равно $\|y-A(x)\|_{L_{1}\left(K_{2}\right)}$, а ближайший элемент - пара $(x, A(x))$. 
Пусть $\left(x_{n}, A\left(x_{n}\right)\right)$ - минимизирующая последовательность для $(x, y)$ в $Y_{A}$. Имеем

$$
\left\|x-x_{n}\right\|_{L_{1}\left(K_{1}\right)}+\left\|y-A\left(x_{n}\right)\right\|_{L_{1}\left(K_{2}\right)} \rightarrow\|y-A(x)\|_{L_{1}\left(K_{2}\right)},
$$

и в тоже время

$$
\begin{aligned}
\| x- & x_{n}\left\|_{L_{1}\left(K_{1}\right)}+\right\| y-A\left(x_{n}\right) \|_{L_{1}\left(K_{2}\right)} \\
& =\left\|x-x_{n}\right\|_{L_{1}\left(K_{1}\right)}+\left\|y-A(x)+A\left(x-x_{n}\right)\right\|_{L_{1}\left(K_{2}\right)} \\
& \geqslant\left\|x-x_{n}\right\|_{L_{1}\left(K_{1}\right)}+\|y-A(x)\|_{L_{1}\left(K_{2}\right)}-\left\|A\left(x-x_{n}\right)\right\|_{L_{1}\left(K_{2}\right)} \\
& \geqslant\|y-A(x)\|_{L_{1}\left(K_{2}\right)}+(1-\|A\|)\left\|x-x_{n}\right\|_{L_{1}\left(K_{1}\right)},
\end{aligned}
$$

откуда в силу неравенства $\|A\|<1$ следует, что $\left\|x-x_{n}\right\| \rightarrow 0$, так что наша минимизирующая последовательность сходится к элементу-паре $(x, A(x))$.

Лемма 1 доказана.

Представим элементы пространства $L_{1}[0,3]$ с обычной мерой Лебега как тройки $(x, y, z)$, где $x, y, z$ - функции, суммируемые на отрезке $[0,1]$. Определим два подпространства

$$
M_{1}=\left\{\left(x, 0, \frac{x}{2}\right): x \in L_{1}[0,1]\right\}, \quad M_{2}=\left\{\left(y, \frac{y}{2}, 0\right): y \in L_{1}[0,1]\right\} .
$$

Из леммы 1 следует, что подпространства $M_{1}$ и $M_{2}$ аппроксимативно компактны в $L_{1}[0,3]$. Очевидно, что алгебраическая сумма $M_{1}+M_{2}$ является замкнутым подпространством.

ТЕОрема 2. Алгебраическая сумма $M=M_{1}+M_{2}$ не является аппроксимативно компактной.

ДокАзАтельство. Докажем для произвольных $x, y \in \mathbb{R}$ неравенство

$$
|x+y-1|+\left|\frac{x}{2}-1\right|+\left|\frac{y}{2}-1\right| \geqslant 1,5 .
$$

Имеем

$$
|x+y-1|+\left|\frac{x}{2}-1\right|+\left|\frac{y}{2}-1\right| \geqslant|x+y-1|+\left|\frac{x+y}{2}-2\right|,
$$

а минимум правой части последнего неравенства достигается либо при $x+y=1$, либо при $x+y=4$. При $x+y=1$ значение правой части равно 1,5 , а при $x+y=4$ оно равно 3. Следовательно, минимум равен 1,5 .

Рассмотрим элемент $w=(1,1,1)$. Заметим, что $w \notin M$. Для любого элемента $f(t)=(x(t)+y(t), y(t) / 2, x(t) / 2)$ из $M$ имеем

$$
\|w-f\|=\int_{0}^{1}\left(|x(t)+y(t)-1|+\left|\frac{y(t)}{2}-1\right|+\left|\frac{x(t)}{2}-1\right|\right) d \mu \geqslant \int_{0}^{1} 1,5 d \mu=1,5,
$$

где $\mu$ - мера Лебега. Таким образом, $\rho(w, M) \geqslant 1,5$.

Построим теперь минимизирующую последовательность для $w$ из $M$, из которой нельзя выбрать сходящуюся подпоследовательность. 
Определим последовательность функций

$$
x_{n}(t)= \begin{cases}0, & 2 i / 2^{n} \leqslant t \leqslant(2 i+1) / 2^{n}, \\ 1, & (2 i+1) / 2^{n}<t<(2 i+2) / 2^{n},\end{cases}
$$

где $i \in \mathbb{Z}, 0 \leqslant i<2^{n-1}, n=1,2, \ldots$. Положим $y_{n}(t)=1-x_{n}(t)$. Тогда элементы $f_{n}(t)=\left(x_{n}(t)+y_{n}(t), y_{n}(t) / 2, x_{n}(t) / 2\right)$ принадлежат $M$ и

$$
\begin{aligned}
\left\|w-f_{n}\right\| & =\int_{0}^{1}\left(\left|1-\left(x_{n}(t)+y_{n}(t)\right)\right|+\left|1-\frac{y_{n}(t)}{2}\right|+\left|1-\frac{x_{n}(t)}{2}\right|\right) d \mu \\
& =\int_{0}^{1}\left(1-\frac{y_{n}(t)}{2}+1-\frac{x_{n}(t)}{2}\right) d \mu=\int_{0}^{1}\left(2-\frac{y_{n}(t)+x_{n}(t)}{2}\right) d \mu \\
& =\int_{0}^{1}(2-0,5) d \mu=1,5
\end{aligned}
$$

т.е. $f_{n}$ является минимизирующей последовательностью для $w$ из $M$. Нетрудно заметить, что $\left\|f_{n}-f_{m}\right\|=1, n \neq m$. Следовательно, из $f_{n}$ нельзя выбрать сходящуюся подпоследовательность, а значит, $M$ не аппроксимативно компактно.

Теорема 2 доказана.

Если отказаться от требования линейности складываемых множеств, то получается более общая

ТЕоремА 3. В любом бесконечномерном банаховом пространстве $X$ существуют два аппроксимативно компактных множества, алгебраическая сумма которых является замкнутым, но не аппроксимативно компактным множеством.

ДокАЗАтЕЛьство. Построим в пространстве $X$ последовательность $\left\{y_{n}\right\}$, удовлетворяющую следующим условиям:

1) $\left\|y_{1}\right\|=1,\left\|y_{n}\right\|=1+\varepsilon_{n}, n=2,3 \ldots$, где $0 \leqslant \varepsilon_{n} \leqslant 1 / 2, \varepsilon_{n} \rightarrow 0, n \rightarrow \infty$;

2) $\rho\left(y_{n+1}, Y_{n}=\left\langle y_{1}, \ldots, y_{n}\right\rangle\right)=1, n=1,2 \ldots$, где $\left\langle y_{1}, \ldots, y_{n}\right\rangle$ обозначает подпространство, порожденное элементами $y_{1}, \ldots, y_{n}$.

Существование такой последовательности доказывается так же, как в п. $1 \S 6$ гл. IV книги [8]. Множество $M_{1}$ определим как $\left\langle y_{1}\right\rangle$. Очевидно, что оно аппроксимативно компактно. Множество $M_{2}$ определим как $\bigcup_{n=2}^{\infty}\left\{\lambda_{n} y_{1}+y_{n}\right\}$, где $\lambda_{n}$ подобраны так, что $\left\|\lambda_{n} y_{1}+y_{n}\right\|=n, n=2,3 \ldots$ (поскольку $\left\|y_{n}\right\|<1,5$, можно подобрать такие $\left.\lambda_{n}\right)$. Поскольку $M_{2}$ состоит из расходящейся по норме последовательности, оно также аппроксимативно компактно. Теперь покажем, что алгебраическая сумма $M=M_{1}+M_{2}$ не является аппроксимативно компактной. Множество $M$ можно представить в виде

$$
M=\bigcup_{n=2}^{\infty}\left\{y_{n}\right\}+\left\langle y_{1}\right\rangle .
$$

В силу того, что $\rho\left(y_{n+1}, Y_{n}\right)=1$, имеем, что $\rho\left(0, y_{n+1}+\left\langle y_{1}\right\rangle\right) \geqslant 1, n=1,2 \ldots$. $\mathrm{C}$ другой стороны, $\left\|y_{n}-0\right\| \rightarrow 1$ по построению $y_{n}$, т.е. последовательность $\left\{y_{n}\right\}$ является минимизирующей для 0 в $M$. В силу условия 2) на последовательность $y_{n}$ из нее нельзя выбрать сходящуюся подпоследовательность, а значит $M$ - не аппроксимативно компактное множество. В то же время множество $M$ замкнуто в силу условия 2) на последовательность $y_{n}$. 
Несложно доказать также следующий факт.

Теорема 4. Если $M_{1}$ компактно, а $M_{2}$ аппроксимативно компактно, то алгебраическая сумма $M=M_{1}+M_{2}$ аппроксимативно компактна.

ДоКАЗАТЕЛЬСТво. Для произвольного элемента $w \notin M$ его минимизирующую последовательность $w_{n}$ представим в виде $w_{n}=x_{n}+y_{n}, x_{n} \in M_{1}, y_{n} \in M_{2}$. В силу компактности $M_{1}$ из $x_{n}$ можно выбрать сходящуюся подпоследовательность $x_{n_{k}} \rightarrow x^{\prime} \in M_{1}$. Тогда, в силу того, что $\left\|w-\left(x_{n_{k}}+y_{n_{k}}\right)\right\| \rightarrow \rho(w, M)$ при $k \rightarrow \infty$, имеем, что $\left\|w-\left(x^{\prime}+y_{n_{k}}\right)\right\| \rightarrow \rho(w, M)$ при $k \rightarrow \infty$. Предположим, что из последовательности $x^{\prime}+y_{n_{k}}$ нельзя выбрать сходящуюся подпоследовательность. В силу аппроксимативной компактности $M_{2}$ это означает, что в ней не содержится подпоследовательности, являющейся минимизирующей последовательностью для $w$ из $M_{2}+x^{\prime}$. Следовательно,

$$
\left\|w-\left(x^{\prime}+y_{n_{k}}\right)\right\|>\rho\left(w, x^{\prime}+M_{2}\right)+\varepsilon>\rho(w, M)+\varepsilon, \quad \varepsilon>0,
$$

при всех $k$. Это противоречит тому, что $\left\|w-\left(x^{\prime}+y_{n_{k}}\right)\right\| \rightarrow \rho(w, M)$ при $k \rightarrow \infty$.

Теорема 4 доказана.

\section{СПИСОК ЦИТИРОВАННОЙ ЛИТЕРАТУРЫ}

[1] Н. В. Ефимов, С. Б. Стечкин, "Аппроксимативная компактность и чебышевские множества", Докл. АН СССР, 140:3 (1961), 522-524.

[2] В.С. Балаганский, Л.П. Власов, "Проблема выпуклости чебышевских множеств", УМН, 51:6 (1996), 125-188.

[3] П. А. Бородин, "Пример ограниченного аппроксимативно компактного множества, не являющегося компактным", УМН, 49:4 (1994), 157-158.

[4] I. Singer, "Some remarks on approximative compactness", Rev. Roumaine Math. Pures Appl., 9:2 (1964), 167-177.

[5] С.В. Конягин, И.Г. Царьков, "Пространства Ефимова-Стечкина", Вестн. Моск. ун-та. Сер. 1. Матем., Мех., 1986, № 5, 20-27.

[6] А. А. Кириллов, А. Д. Гвишиани, Теоремы и задачи функционального анализа, Наука, M., 1988.

[7] П.А. Бородин, "О линейности оператора метрического проектирования на чебышевские подпространства в пространствах $L_{1}$ и $C$ ", Матем. заметки, 63:6 (1998), $812-820$.

[8] А. Н. Колмогоров, С. В. Фомин, Элементы теории функиий и функиионального анализа, Наука, М., 1989.

И. А. Пятышев

Московский государственный университет

им. М. В. Ломоносова

E-mail: pyatyshoff@mail.ru
Поступило

05.07.2006

Исправленный вариант 16.04.2007 